\title{
Perceiving object dangerousness: an escape from pain?
}

\author{
Filomena Anelli • Mariagrazia Ranzini • \\ Roberto Nicoletti · Anna M. Borghi
}

Received: 10 October 2012 / Accepted: 14 May 2013 / Published online: 7 June 2013

(C) Springer-Verlag Berlin Heidelberg 2013

\begin{abstract}
A variety of studies showed that participants are facilitated when responding to graspable objects, while it has not been fully investigated what happens during interactions with graspable objects that are potentially dangerous. The present study focuses on the mechanisms underlying the processing of dangerous objects. In two experiments, we adopted a paradigm that has never been employed in this context, a bisection task. The line was flanked by objects belonging to different categories. We explored the sensitivity to the distinction between neutral and dangerous objects, by measuring whether the performance was biased toward a specific object category. In Experiment 1 both teenagers and adults bisected lines flanked by dangerous and neutral graspable objects, and they misperceived the line midpoint toward the neutral graspable object or, stated differently, on the opposite side of the dangerous graspable object. In Experiment 2 adults bisected lines flanked
\end{abstract}

\section{F. Anelli $(\varangle) \cdot R$. Nicoletti}

Department of Philosophy and Communication,

University of Bologna, via Azzo Gardino, 23,

40122 Bologna, Italy

e-mail: filomena.anelli@unibo.it

F. Anelli

Department of Education Sciences, University of Bologna,

Bologna, Italy

M. Ranzini

FNRS, CRCN, Université Libre de Bruxelles (ULB),

Bruxelles, Belgium

A. M. Borghi

Department of Psychology, University of Bologna, Bologna, Italy

A. M. Borghi

Institute of Cognitive Sciences and Technologies,

National Research Council, Rome, Italy by dangerous and neutral objects matched on graspability (both graspable and ungraspable, Experiment 2a), or by graspable and ungraspable objects matched on dangerousness (both neutral and dangerous, Experiment 2b). Results confirmed the finding of Experiment 1, but also indicated that participants misperceived the line midpoint toward the ungraspable object when it was presented, being it dangerous or not. This evidence demonstrated sensitivity to object dangerousness maintained across lifespan. The emergence of aversive affordances evoked by dangerous graspable objects strenghtens the importance to consider graspability in the investigation of dangerous objects. Possible neural mechanisms involved in the processing of dangerous graspable objects are discussed.

Keywords Line bisection task - Affordances . Dangerous objects · Graspability · Motor simulation . Embodied cognition $\cdot$ Canonical neurons

\section{Introduction}

An important human ability is to respond properly to objects in the environment, for example discriminating objects that can be useful from objects that can cause danger. Gibson (1979) used the term "affordances" to refer to properties of the environment providing the observers with practical opportunities that they are able to perceive and use. This term refers to the relationship between the organisms' motor repertoire, their goals, and the environment. Recent studies on human-object interaction started from this first conceptualization of affordances, but also slightly departed from it: for example, Ellis and Tucker (2000) proposed to use the term "micro-affordances" to refer to the activation of specific motor components (e.g. reaching or 
grasping motor response) during object observation. The results of a variety of studies on the processing of object and action highlight that this activation would re-enact perception-action brain assemblies, formed during previous experience (for reviews, see also Martin 2007; Bub and Masson 2010; Borghi et al. 2012). Therefore, the representation of dangerous objects may also rely on previous sensory-motor experiences.

Several studies have shown that perceiving graspable objects induces compatibility effects: the kinematic parameters and speed of action execution are influenced by the relation between object properties (e.g. size: Castiello 2003; Edwards et al. 2003; orientation: Craighero et al. 1998; consistence: Anelli et al. 2010) and action properties (e.g. side of response: Tucker and Ellis 1998 and kind of grip: Ellis and Tucker 2000; Tucker and Ellis 2001, 2004). Moreover, neuroimaging studies have shown that the neural activity in motor-related brain areas increases during object observation (Chao and Martin 2000; Creem-Regehr and Lee 2005; Gerlach et al. 2002; Grafton et al. 1997; Johnson-Frey 2004). Again, a planned or executed action can bias attention, inducing facilitation in detecting targets whose properties are compatible with the action (Craighero et al. 1999; Symes et al. 2008, 2010). Importantly, the execution of an action is not necessary to induce attentional biases, in that observing a hand acting toward an object is sufficient to direct attention (Fischer et al. 2008; Ranzini et al. 2011a; Fagioli et al. 2007). These findings converge in suggesting that object and action representations communicate bidirectionally (Goslin et al. 2012).

While much evidence shows that participants are facilitated when responding to graspable objects (i.e. objects that evoke the affordance of grasping), what happens when interacting with potentially dangerous objects has not been fully investigated. The most part of studies that have made use of dangerous or painful stimuli pertain to the domain of pain investigation. These studies were conducted by means of different techniques: behavioural measures as reaction times (e.g. Morrison et al. 2007b), brain imaging (e.g. Singer et al. 2004; Morrison et al. 2007a), or transcranial magnetic stimulation (TMS) paradigms (e.g. Avenanti et al. 2005, 2006, 2010). For instance, in a series of TMS studies on empathy for pain participants observed images of a needle, which was inserted into a model's hand (e.g. Avenanti et al. 2005, 2006, 2010). Results showed that the motor evoked potential (MEP) amplitude was modulated by pain observation. This painrelated inhibition was linked both to measures of the model's sensory qualities of the pain (such as the pain intensity) and of the state sensory empathy (e.g. Avenanti et al. 2005), suggesting the activation of motor resonance mechanisms.

Recent behavioural studies aimed at distinguishing the effects evoked by the presentation of the graspable objects (dangerous vs. neutral) and the motor resonance effects elicited while observing hands in potential interaction with them (Anelli et al. 2012a, b). These studies investigated the sensitivity to the distinction between graspable neutral and dangerous objects in school-age children and adults, as well as the possible motor resonance induced by the observation of others' actions. To this aim, a priming paradigm was used: a hand prime or a control object prime was followed by a neutral graspable or dangerous object. Participants were required to categorize it into artefact or natural object by pressing a different key. In both adults and children, neutral graspable objects facilitated the motor response. In contrast, the response to dangerous objects was slower than to neutral ones, suggesting that dangerous objects evoked aversive affordances, generating an interference effect. Moreover, both children and adults were sensitive to the distinction between biological versus non-biological hands, indicating that motor resonance mechanisms were at play only during biological hands observation, and that the higher the motor resonance evoked by biological hands, the stronger the inhibition obtained with dangerous objects. More relevant to our aims, dangerous objects produced a slowdown of responses and an interference effect. These findings are in line with a body of studies investigating the ability to detect fear-relevant and fear-irrelevant stimuli by means of a visual search paradigm (e.g. Blanchette 2006). These studies have revealed a threat-superiority effect, namely threatening objects were detected faster and more efficiently than non-threatening ones.

The behavioural studies described so far showed that human motor responses are influenced by object dangerousness, but objects were always primed by hands in potential interaction with them. In contrast, the present work investigates neutral and dangerous objects processing when no agent is interacting with them, thus allowing to investigate the dangerousness perception independently from the observation of others' actions. In fact, it is important to understand how humans represent dangerous or painful objects, which mechanisms are at play while interacting with them, and which relevant variables (such as developmental stages or relevant experiences) may influence dangerous object representations (for another study on neutral and dangerous objects processing when no agent was shown, see Anelli et al. under review).

While most of the studies on affordances employed a stimulus-response compatibility paradigm (SRC), in the present work we explore whether and to what extent performance is influenced by object dangerousness with a paradigm that has never been employed in this context, that is the cued line bisection task. In this task, participants are required to bisect a line flanked by two different irrelevant cues at the extremities. So, one of the novelties of the present research is the chosen paradigm, the cued line bisection task, in which the role of cues is irrelevant for the 
execution of the task. Typically, the line bisection task is used as research instrument to study visuospatial attention in healthy individuals and as neuropsychological diagnostic instrument, for example with patients with visuospatial neglect or hemianopia (e.g. Barton et al. 1998; Bisiach et al. 1976; Kerkhoff and Bucher 2008; Schenkenberg et al. 1980). Typically, healthy individuals show a leftward bias in this task, named pseudoneglect, revealing visuospatial asymmetries in the attentional system (Jewell and McCourt 2000). More relevant to our aim, this paradigm has proven to be useful to study symbolic cueing processing through the analysis of bisection biases (see Fischer 2001). For example, Ranzini et al. (2011a) investigated action- and object-related motor cueing effects, by means of a handcued line bisection task. Participants were presented with a line (thin vs. thick line) flanked with images of hands (biological vs. non-biological hand) representing different actions (power vs. precision grip). Performance was biased toward the action more compatible with the object (power grip-thick line and precision grip-thin line), showing that the bisection paradigm was sensitive to action- and objectrelated motor cueing. Importantly, this effect was reduced or absent with non-biological hands, suggesting that motor resonance mechanisms underlie bisection performance with hand cueing. Here, participants were presented with a line flanked by two pictures of objects, with the idea that objects observation would evoke affordances, which in turn would bias bisection performance. The line was flanked by objects-not by hands pictures as in Ranzini et al. (2011a) — since our interest lied in investigating the object affordance effect independently from the effect evoked by the presentation of the hand.

We conducted two experiments, where the line stimulus was flanked by images of objects belonging to different categories. In Experiment 1, both teenagers and adults bisected lines flanked by a pair of stimuli representing a dangerous and a neutral graspable object. In Experiment 2 , adults bisected lines flanked by a pair of stimuli representing a dangerous and a neutral object matched on graspability (both graspable and ungraspable, Experiment 2a), or representing a graspable and an ungraspable object matched on dangerousness (both neutral and dangerous, Experiment $2 b)$. We explored the sensitivity to the distinction between neutral and dangerous objects, by measuring whether the performance was biased toward/away from a specific object category. On the basis of aforementioned evidence, we hypothesized to observe in Experiment 1 a bias in the opposite direction to the dangerous object and, as a consequence, toward the neutral graspable object. We also investigated how the sensitivity to aversive affordances differed at different life stages, by testing performance of both teenagers and adults. As mentioned above, the ability to distinguish dangerous and neutral objects emerges quite early in development, as it might be crucial from an adaptive point of view (Anelli et al. 2012a, b). However, it is possible that this ability increases with age: we tested this hypothesis by comparing teenagers and adults performance.

Finally, in Experiment 2, we investigated whether the predicted bias in the opposite direction to the dangerous object was due to an affordance effect caused by neutral graspable objects (hypothesis 1) or to a withdrawal effect caused by dangerous graspable objects (hypothesis 2). To disentangle this point, we adopted stimuli matched on graspability but differing on dangerousness (Experiment 2a) or matched on dangerousness but differing on graspability (Experiment 2b). Following hypothesis 1, we would expect a bias toward the neutral graspable object (in Experiment $2 \mathrm{a}$ with graspable objects; in Experiment $2 \mathrm{~b}$ with neutral objects). On the other hand, following hypothesis 2 , we would expect a bias in the opposite direction of the dangerous graspable object side (in Experiment 2a with graspable objects; in Experiment $2 \mathrm{~b}$ with dangerous objects). We do not have clear predictions about ungraspable objects, except that they should not evoke grasping affordances.

\section{Experiment 1: neutral graspable versus dangerous objects in teenagers and adults}

Method

Participants

Fourteen teenagers ( 4 males and 10 females; 12 years old) and twelve undergraduate students from the University of Bologna ( 6 males and 6 females; mean age: 21 years, range: 19-26) took part in the experiment. All subjects were righthanded and had normal or corrected-to-normal vision. All were naive as to the purpose of the experiment and they or their parents, as for teenagers, gave informed consent.

\section{Materials and procedure}

Participants sat in front of a 17 -inc. colour monitor (the eye-to-screen distance was approximately $50 \mathrm{~cm}$ ). E-Prime 1.1 software was used.

The task was a computerized version of the line bisection task. Participants were required to indicate the midpoint of a line flanked by two pictures of objects (object-line distance: $4 \mathrm{px}, 0.1 \mathrm{~cm}$ ). Participants indicated the midpoint with the help of a mouse cursor (a vertical arrow, size: $17 \times 7 \mathrm{px}, 0.5 \times 0.2 \mathrm{~cm}$ ). The arrow cursor shifted only horizontally under the line. The line was centrally presented on the horizontal axis, but its vertical position (centre, up, or down from the screen centre) and the arrow cursor initial position (left or right under the line) were 
Table 1 Experimental stimuli

\begin{tabular}{|c|c|c|c|}
\hline $\begin{array}{l}\text { Neutral } \\
\text { graspable } \\
\text { objects (exp 1, } \\
2 \mathrm{a} \text {, and } 2 \mathrm{~b})\end{array}$ & $\begin{array}{l}\text { Dangerous } \\
\text { graspable objects } \\
(\exp 1,2 \mathrm{a}, \\
\text { and } 2 \mathrm{~b})\end{array}$ & $\begin{array}{l}\text { Neutral } \\
\text { ungraspable } \\
\text { objects (exp 2a } \\
\text { and 2b) }\end{array}$ & $\begin{array}{l}\text { Dangerous } \\
\text { ungraspable } \\
\text { objects (exp } \\
2 \mathrm{a} \text { and } 2 \mathrm{~b})\end{array}$ \\
\hline \multicolumn{4}{|l|}{ Natural } \\
\hline Cat & Porcupine & Elephant & Crocodile \\
\hline Chick & Scorpion & Dolphin & Shark \\
\hline Plant & Cactus & Tree & Prickly pear \\
\hline Tomato & Husk & Mountain & Volcano \\
\hline \multicolumn{4}{|l|}{ Artefact } \\
\hline Bulb & Broken bulb & Bell & Atomic bomb \\
\hline Glass & Broken glass & Skyscraper & $\begin{array}{l}\text { Collapsing } \\
\text { skyscraper }\end{array}$ \\
\hline $\begin{array}{l}\text { Lighted } \\
\text { out match }\end{array}$ & Lighted match & Semaphore & $\begin{array}{l}\text { Warning road } \\
\text { sign }\end{array}$ \\
\hline Spoon & Knife & Home & $\begin{array}{l}\text { Collapsing } \\
\text { home }\end{array}$ \\
\hline
\end{tabular}

randomized across trials and not considered as variables of interest. Two line lengths were presented (short: $288 \mathrm{px}$, $7.6 \mathrm{~cm}$; long: $432 \mathrm{px}, 11.4 \mathrm{~cm}$ ).

The object flankers consisted of sixteen black and white pictures of common graspable objects, half of the objects were neutral (mean pixel $125 \times 166$, mean $\mathrm{cm} 3.3 \times 4.4$ ), for example a tomato, and half dangerous (mean pixel $120 \times 165$, mean $\mathrm{cm} 3.2 \times 4.4$ ), for example a cactus (see Table 1). The set of objects was the same used in other studies (Anelli et al. 2012a, b), in which we asked an independent group of forty-three participants to rate on a five-points Likert scale objects dangerousness.

In each trial, the object flanker pairs could belong to two conditions depending on the objects positions: neutral left + dangerous right (Neutral Dangerous, ND) or dangerous left + neutral right (Dangerous Neutral, DN).

Instructions indicated the presence of a line flanked by two objects, emphasizing the importance to correctly indicate the line midpoint without considering the objects.

The experiment consisted of one practice block of 16 trials and one experimental block of 96 trials. In each block, half of the trails belonged to the ND condition and half to the DN condition. Objects position conditions (ND, DN) and line lengths (long, short) were presented in a randomized order, for a total of 112 trials.

Each trial began with a white screen displayed for $100 \mathrm{~ms}$. Then, a line flanked by two objects was shown, followed after $100 \mathrm{~ms}$ by the arrow cursor. Stimuli remained on the centre of the screen until response. Then, a white screen was presented for $1,000 \mathrm{~ms}$, before the next trial (see Fig. 1).

At the end of the experiment, participants rated the dangerousness of the sixteen objects presented during the experiment on a five-point Likert scale (with $1=$ not dangerous and potentially painful object, and $5=$ extremely dangerous and potentially painful object).

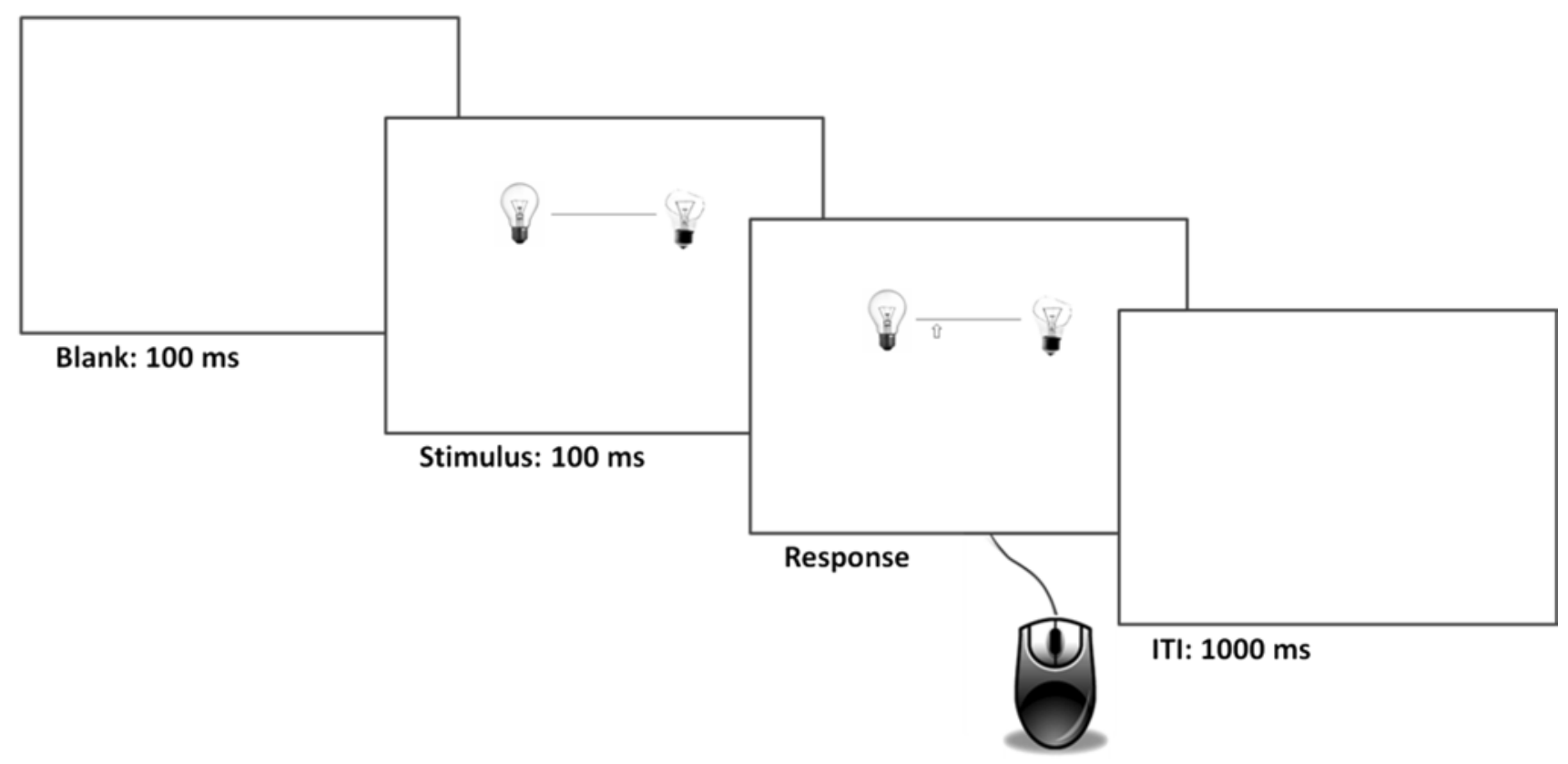

Fig. 1 Design of the experiments. In the two experiments, participants were required to indicate the midpoint of a line flanked by two pictures of objects. Each trial started with a white screen displayed for $100 \mathrm{~ms}$. Then, a line flanked by two objects was shown followed after $100 \mathrm{~ms}$ by the arrow cursor under the line. The stimuli remained on the centre of the screen until a response had been made. After stimulus offset, a white screen was presented for $1,000 \mathrm{~ms}$, and then the next trial began 
The experiment consisted of a 20-min session, after which participants were informed about the aims of the experiment.

\section{Scoring and analysis}

Our dependent variable was the accuracy in the bisection task, obtained subtracting the real line midpoint (the line centre) from the subjective line midpoint (i.e. participant's bisection). In this way, accuracy is referred to the line centre, and positive values correspond to rightward bias while negative values correspond to leftward bias. Accuracy is expressed in pixel, similarly to previous studies adopting this paradigm (Ranzini et al. 2011a; Ranzini and Girelli 2012).

Statistical analyses were conducted by means of an ANOVA on accuracy, with three factors: Group (teenagers and adults) as between-subjects factor, and Objects Order (ND and DN) and Line Length (long and short) as withinsubjects factors.

In addition, an ANOVA on dangerousness ratings was carried out, with three between-subjects factors: Group (teenagers and adults), Typology (neutral and dangerous), and Category (artefact and natural).

\section{Results}

The accuracy analysis revealed two significant main effects, Objects Order $\left[F(1,24)=14.1, M S e=3.02, n_{p}^{2}=0.37\right.$, $p=.001]$ and Group $[F(1,24)=5.9, \mathrm{MSe}=64.9$, $\left.n_{p}^{2}=0.20, p=.02\right]$. There was a larger leftward bias in ND condition (mean $=-2.5, \mathrm{SEM}=0.8$ ) than in DN condition (mean $=-1.2$, SEM $=0.8$ ), (Fig. 2). Post hoc analyses revealed that the leftward bias was significant only in the ND condition ( $t$ test vs. 0 : ND, $t(25)=-2.7, p<0.05$; $\mathrm{DN}, t(25)=-1.1, p>0.3)$. In addition, only adults showed a leftward bias (adults mean $=-3.7, \mathrm{SEM}=1.2$; teenagers mean $=0.1, \mathrm{SEM}=1.1$ ). Post hoc analyses revealed that the leftward bias was significant only in adults ( $t$ test vs. 0 : adults, $t(11)=-4.2, p<0.01$; teenagers, $t(13)=0.9$, $p>0.9)$. There was no other significant main effect or interaction ( $p \mathrm{~s}>0.05)$.

The rating analysis revealed the main effect of Typology $[F(1,24)=187.1, M S E=0.2, p<.001]$ showing a significant difference between neutral (mean $=1.4$ ) and dangerous objects (mean $=3.4)$. There was no other significant main effect or interaction ( $p$ s $>0.05)$.

\section{Discussion}

Experiment 1 demonstrated that bisection performance was influenced by the type of flanker objects: participants significantly shifted the midpoint toward the neutral object. This pattern of results might suggest that the neutral
Experiment 1: Neutral and Dangerous objects in teenagers and adults

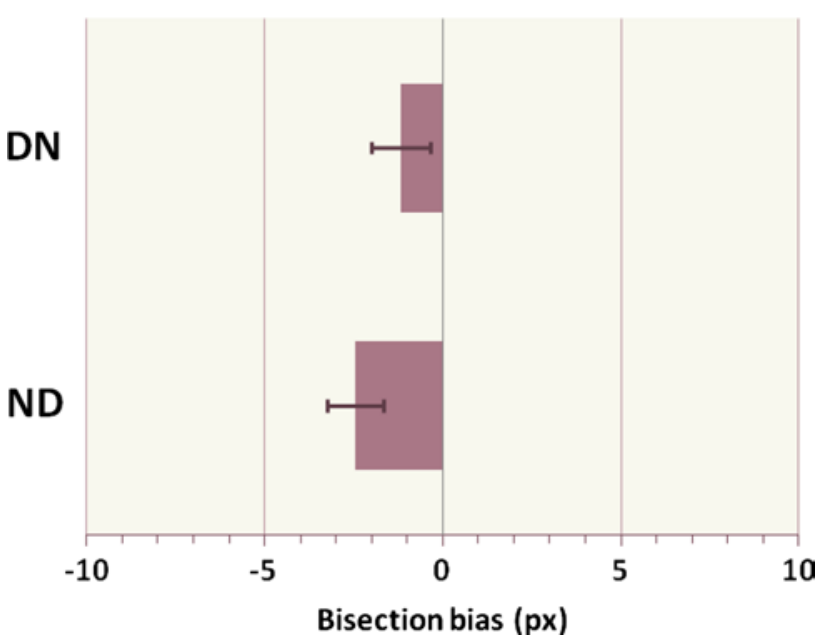

Fig. 2 Significant Objects Order effect for the bisection bias in Experiment 1. Values are in pixels and bars are SEM. Negative and positive values correspond to shifts toward the left or right from the centre, respectively. Participants (teenagers and adults) shifted the midpoint toward the neutral object when the line was flanked by dangerous and neutral objects

graspable object evokes an affordance effect, biasing the motor response toward it. Alternatively, or in addition, the dangerous graspable object might generate avoidance/ repulsion effects, thus inducing an "escape" from it.

Despite a classical leftward bias characterized the adult group only (differently to the decreasing in pseudoneglect in function of age observed in previous studies: see Jewell and McCourt 2000), adults' bias due to object typology did not significantly differ from the teenagers' one. Therefore, teenagers and adults were similarly sensitive to the difference between dangerous and neutral objects.

\section{Experiment 2: dangerousness and graspability in adults}

Experiment 1 showed that, when required to indicate the midpoint of a line flanked by pictures of neutral and dangerous graspable objects, younger and adult participants bisected the line toward the neutral object. This demonstrates the human ability to automatically discriminate object properties-here object affordances, whether of graspability or avoidance-even when the object is not relevant for the task. However, this finding can either originate from the neutral object, inducing graspable affordances, or it can be an "escape" from the dangerous one, inducing aversive affordances. Experiment 2 was conducted to clarify whether the previous result was linked to an affordance effect evoked by the neutral graspable object or to an avoidance of the dangerous graspable one, by presenting 
ungraspable objects that, by their nature, should not evoke affordances linked to graspability. The line was flanked by two objects matched on graspability and differing on dangerousness (Experiment 2a), or matched on dangerousness and differing on graspability (Experiment $2 b$ ). If the effect observed in Experiment 1 was due to the tendency to reach the graspable object, we would observe an effect toward it also when coupled with an ungraspable one. On the other hand, if it was due to an escape from the dangerous graspable object, we would observe a bias opposed to it also when coupled with an ungraspable one.

Due to the lack of influence of different age classes reported in Experiment 1, only adults participated to Experiment 2.

\section{Experiment 2a}

Method

\section{Participants}

Twelve undergraduate students from the University of Bologna ( 3 males and 9 females) with a mean age of 19.8 years (range: 19-23) took part in Experiment 2a for course credits. All subjects had normal or corrected-to-normal vision. All were naive as to the purpose of the experiment and gave informed consent.

\section{Materials and procedure}

Apparatus, stimuli, and procedure were the same of Experiment 1 except for the following. Since Experiment 2a was focused on dangerousness, objects differed for their dangerousness, but were matched on graspability. Thus, we compared neutral graspable with dangerous graspable objects and neutral ungraspable with dangerous ungraspable objects.

The object flankers consisted of thirty-two black and white pictures of objects. Sixteen objects were the same ones employed in Experiment 1. The other sixteen objects were new, half were neutral ungraspable objects (mean pixel $122 \times 162$, mean cm $3.2 \times 4.3$ ), for example a mountain, and half were dangerous ungraspable objects (mean 123 pixel $\times 163$, mean $\mathrm{cm} 3.3 \times 4.3$ ), for example a volcano (see Table 1).

In each trial, the object flanker pairs could belong to four conditions depending on the objects positions: left neutral + right dangerous graspable (NDG) or left dangerous + right neutral graspable (DNG) and left neutral + right dangerous ungraspable (NDU) or left dangerous + right neutral ungraspable (DNU).

The experiment consisted of one practice block of 16 trials and one experimental block of 96 trials (for a total of 112 trials, as in Experiment 1). Only long lines were presented, since in Experiment 1 no differences emerged between short and long lines.

At the end of the experiment, participants were required to rate both the dangerousness and the graspability of the thirty-two objects presented during the experiment on a five-points Likert scale (rating on dangerousness: $1=$ not dangerous and potentially painful object and $5=$ extremely dangerous and potentially painful object; rating on graspability: $1=$ ungraspable object and $5=$ graspable object). Object graspability was evaluated on the basis of whether it was possible or not to lift the object with the hands and move it from one place to another (for a similar procedure, see Ranzini et al. 2011b).

The experiment consisted of a 25-min session, and then participants were informed about the aims of the experiment.

\section{Scoring and analysis}

We analysed the accuracy as in Experiment 1.

Statistical analyses were conducted by means of an ANOVA on accuracy, with two factors: Typology (dangerous and neutral) and Objects Order (graspable left and ungraspable right/graspable right and ungraspable left) as within-subjects factors.

In addition, for each block, two ANOVAs on ratings concerning dangerousness and graspability were carried out, with the between-subjects factor Typology (neutral and dangerous) or Graspability (graspable and ungraspable), respectively.

\section{Results}

The accuracy analysis showed a significant interaction Objects Order $\times$ Typology $[F(1,11)=37.5$, MSe $=0.8$, $\left.n_{p}^{2}=0.8, p<.001\right]$, (Fig. 3, panel a). With graspable objects, participants indicated the midpoint of the line toward the neutral object/far from the dangerous one [NDG condition: mean $=-3.2, \mathrm{SEM}=1.7 ; \mathrm{DNG}$ condition: mean $=-1.8$, $\mathrm{SEM}=1.8 ; t(11)=-3.4, p<0.01]$, exactly as observed in Experiment 1. With ungraspable objects, instead, participants indicated the midpoint of the line toward the dangerous object/far from the neutral one [NDU condition: mean $=-2.7$, SEM $=1.8$; DNU condition: mean $=-4.2$, $\mathrm{SEM}=1.7 ; t(11)=3.2, p<0.01]$. The leftward bias was significant only in the DNU condition $[t$ test vs. 0 : $t(11)=-2.4, p<0.05$; in the NDG condition: $p=0.08]$.

The ratings analysis on dangerousness revealed the main effect of Typology $[F(1,30)=48.4, M S E=0.8, p<.001]$, with a significant difference between neutral $($ mean $=1.4)$ and dangerous objects (mean $=3.6$ ).

The rating analysis on graspability revealed the main effect of Graspability $[F(1,30)=119.1, M S E=0.5$, 
Fig. 3 Significant interaction Object Order $\times$ Typology for the bisection bias in Experiment 2. Values are in pixels and bars are SEM. Negative and positive values correspond to shifts toward the left or right from the centre, respectively.

a Significant interaction between Typology and Objects Order for the bisection bias in Experiment 2a. Participants shifted the midpoint toward the neutral graspable object, but also toward the dangerous ungraspable one. b Significant interaction between Typology and Objects Order for the bisection bias in Experiment $2 \mathrm{~b}$. Participants shifted the midpoint toward the ungraspable object, but this effect was larger with dangerous stimuli
Panel A

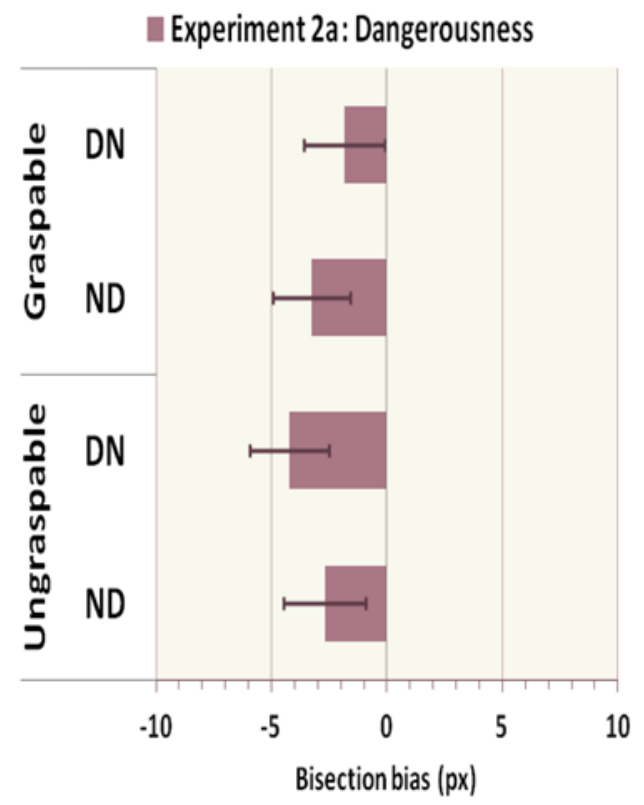

Panel B

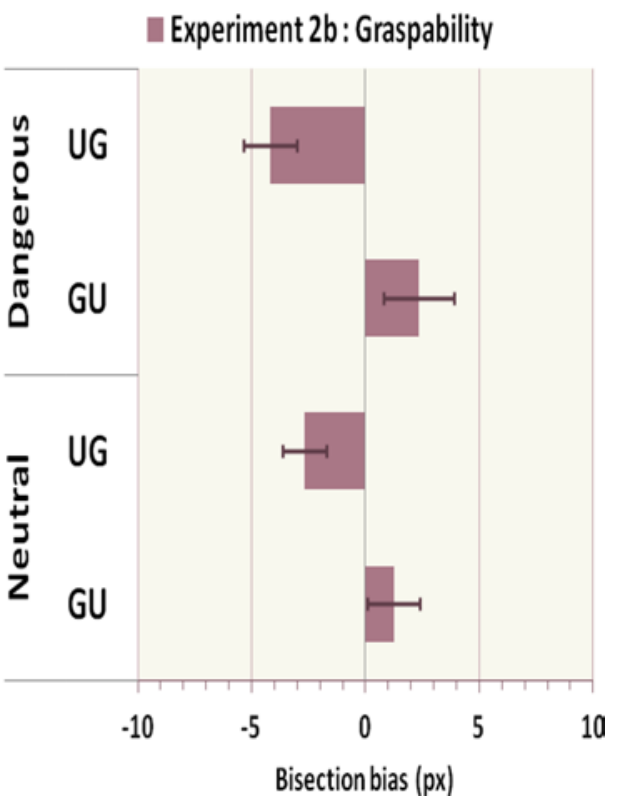

$p<.001$ ], with a significant difference between graspable $($ mean $=4.2)$ and ungraspable objects $($ mean $=1.4)$.

\section{Discussion}

Experiment 2a showed two interesting results. First, with graspable objects, participants shifted the midpoint toward the neutral objects and far from the dangerous ones, confirming the finding of Experiment 1. As in Experiment 1, the results do not allow clarifying whether the effect is due to an affordance effect induced by the neutral object or an avoidance effect induced by the dangerous one. Experiment $2 b$ will give further hints on this issue. Second, data on ungraspable objects extended the previous ones revealing the opposite pattern of results, since in this case participants indicated the midpoint of the line toward the dangerous object and far from the neutral one. We interpret this last effect as a pure effect of attention toward the dangerous stimulus (e.g. Blanchette 2006) when no grasping affordance is involved.

\section{Experiment 2b}

Method

\section{Participants}

Twelve undergraduate students from the University of Bologna ( 1 male and 11 females) with a mean age of 20.3 years (range: 19-24) took part in Experiment $2 b$ for course credits. All subjects had normal or corrected-to-normal vision.
All were naive as to the purpose of the experiment and gave informed consent.

\section{Materials and procedure}

Apparatus, stimuli, and procedure were the same of Experiment 1 except for the following. Since Experiment $2 b$ was focused on graspability, objects differed for their graspability, but were matched on dangerousness. We compared neutral graspable with neutral ungraspable objects and dangerous graspable with dangerous ungraspable objects.

In each trial the object flanker pairs could belong to four conditions depending on the objects positions: left graspable + right ungraspable neutral (GUN) or left ungraspable + right graspable neutral (UGN) and left graspable + right ungraspable dangerous (GUD) or left ungraspable + right graspable dangerous (UGD).

\section{Scoring and analysis}

We analysed the accuracy as in Experiment $2 \mathrm{a}$ except for the following.

Statistical analyses were conducted by means of an ANOVA on accuracy, with two factors: Typology (graspable and ungraspable) and Objects Order (dangerous left and neutral right/dangerous right and neutral left) as within-subjects factors.

Results

The accuracy analysis showed the main effect of Objects $\operatorname{Order}\left[F(1,11)=75.2, \mathrm{MSe}=4.4, n_{p}^{2}=0.9, p<.001\right]$, 
with a bias toward the ungraspable object (graspableungraspable condition: mean $=1.8, \mathrm{SEM}=1.3$; ungraspable-graspable condition: mean $=-3.4$, SEM $=1.0$ ). Importantly, the interaction Objects Order $\times$ Typology $\left[F(1,11)=24.3, M S e=0.8, n_{p}^{2}=0.7, p<.001\right]$ was significant (Fig. 3, panel b). The interaction indicated that the effect toward the ungraspable object was larger with dangerous objects [GUD condition: mean $=2.3, \mathrm{SEM}=1.6$; UGD condition: mean $=-4.2, \mathrm{SEM}=1.2 ; t(11)=8.9$, $p<0.001$ ] than with neutral ones [GUN condition: mean $=1.3, \mathrm{SEM}=1.2 ; \mathrm{UGN}$ condition: mean $=-2.7$, $\mathrm{SEM}=1.0 ; t(11)=6.9, p<0.001]$. The left bias was significantly different from 0 both in the UGN $[t(11)=-2.8$, $p<0.05]$ and in the UGD conditions $[t(11)=-3.6$, $p<0.01]$.

The ratings analysis on dangerousness revealed the main effect of Typology $[F(1,30)=42.4, M S E=0.8, p<.001]$. As in Experiment 2a, this result showed a significant difference between neutral $($ mean $=1.5)$ and dangerous objects $($ mean $=3.6)$.

The rating analysis on graspability revealed the main effect of Graspability $[F(1,30)=173.6, M S E=0.4$, $p<.001]$, with a significant difference between graspable $($ mean $=4.2)$ and ungraspable objects $($ mean $=1.4)$.

\section{Discussion}

Experiment $2 \mathrm{~b}$ demonstrated that when objects differed in graspability, participants shifted significantly the midpoint toward the ungraspable object rather than toward the graspable one. Albeit we do not have a clear understanding of the reasons underlying the effect of attraction toward the ungraspable neutral object, it is important to underline that this effect is not supporting the view that the bias toward the dangerous object when both are graspable is due to an effect of affordance toward the neutral object (hypothesis 1). On the contrary, together with results of Experiment 2a, this finding is in line with the view that dangerous graspable objects may evoke aversive affordances (hypothesis 2). The fact that the bias toward the ungraspable object was larger when the objects were dangerous rather than neutral can be due to a conjoint effect of avoidance of graspable dangerous object and attraction toward the ungraspable dangerous one.

\section{General discussion}

The present study investigated the mechanisms underlining the processing of dangerous objects. In two experiments, we measured participants' sensitivity to the distinction between neutral and dangerous objects during cued line bisection (e.g. Ranzini et al. 2011a, b). In Experiment
1, the line was flanked by neutral and dangerous graspable objects. Both teenagers and adults performed the task allowing us to investigate whether the ability to distinguish dangerous and neutral objects increases with age. The bisection bias was considered as an indicator of underlying mechanisms of objects processing.

Firstly, we found that participants systematically misbisected the line toward the neutral graspable object in Experiment 1, that is, in the opposite direction of the dangerous object side. This effect was similarly observed in teenagers and adults. This result demonstrates that participants were sensitive to dangerous objects and that this sensitivity is maintained across lifespan, possibly as it is necessary for survival (see also Anelli et al. 2012a, b). These results are in line with a large body of evidence indicating that perceiving danger modulates/involves visual attention. For instance, in visual search tasks, dangerous stimuli are more efficiently detected than neutral ones (threat-superiority effect, e.g., Blanchette 2006; Öhman et al. 2001). The present study is in line with these studies: first, it confirms that threat modulates the allocation of visuospatial attention, as the orienting of attention is crucially involved while performing the bisection task (see Toba et al. 2011). Secondly, we observed danger sensitivity in a task not requiring SRC and where the object stimuli were completely task irrelevant (i.e. they did not need to be processed to perform line bisection), suggesting a certain degree of automaticity in the processing of danger (Öhman et al. 2001).

Moreover, differently from previous behavioural and TMS studies (e.g. Anelli et al. 2012a, b; Avenanti et al. 2005; Morrison et al. 2007b), the objects were not presented in interaction with a hand, permitting us to attribute the observed bias to a pure effect of object processing and not to the presence of motor resonance mechanisms.

The systematic misplacement of the subjective line midpoint toward the neutral graspable object/against the dangerous object, however, had two possible explanations: it could be due to the activation of graspable objects affordances; alternatively, it could be due to an aversive affordance inducing "escaping" from the dangerous objects.

Experiment 2 clarified whether the effect obtained in Experiment 1 was due to affordances activation by neutral graspable objects or to interference effects generated by dangerous objects. In Experiment 2a, lines were flanked by objects matched on graspability (graspable or ungraspable) and differing on dangerousness (neutral or dangerous). In Experiment $2 b$, lines were flanked by objects matched on dangerousness (neutral or dangerous) and differing on graspability (graspable or ungraspable). Results of Experiment $2 \mathrm{a}$ confirmed the finding of Experiment 1 by revealing that, when participants were faced with graspable objects, they bisected the line relatively toward the neutral 
object, and thus far from the dangerous one. However, data highlighted new and unexpected evidence as for ungraspable objects: participants shifted the subjective midpoint toward the dangerous object. Notice that the paradigm here adopted involved the planning and execution of a precise hand action (i.e. doing a mark on the line). In this sense, the effects of graspable stimuli are in line with an interpretation that takes into account the interplay between object affordance, attention, and action planning and execution (Ranzini et al. 2011a, b). However, with ungraspable stimuli, the manual task seemed to be less influenced by the objects' dangerousness given that, by their nature, ungraspable objects should not evoke affordances related to graspability. The effect of ungraspable dangerous stimuli was opposite to the one obtained with graspable ones, probably due to attentional factors. Indeed, an ungraspable dangerous object seemed to be more salient than an ungraspable neutral one, capturing the participants' attention and thus inducing the bisection bias (in line with the attentional account, for example, Öhman et al. 2001). Importantly, Experiment $2 \mathrm{~b}$ showed that ungraspable objects were more attractive than graspable ones, either dangerous or neutral, even if this effect was larger with dangerous stimuli than with neutral ones. Albeit we do not have a clear explanation of the bias toward the ungraspable object when coupled with graspable ones, this effect favours the idea that dangerous graspable objects evoke some kind of avoidance effect (Anelli et al. 2012a, b), which in turn cause an "escape". This allows excluding the hypothesis 1 . As graspable objects trigger the affordance of grasping, participants moved away from the object when perceived it as graspable and potentially dangerous.

Moreover, the bias toward dangerous stimuli when ungraspable underlines the importance of taking into account the dimension of graspability when investigating the processing of danger, as opposite results can emerge depending on this dimension.

The paradigm we chose allows us to speculate about the possible neural mechanisms underlying the processing of neutral and dangerous graspable objects. Previous studies on pain investigated the relationship between a hand and an object (e.g. Anelli et al. 2012a, b; Avenanti et al. 2005; Morrison et al. 2007b), highlighting the role of the mirror neuron system (i.e. neurons involved in both the agent's own actions and the visual observation of such actions performed by others; for a review, see Rizzolatti and Craighero 2004) as the possible underlying basis of a resonant mechanism activated while observing hands interacting with painful stimuli. In the present research, we presented only objects. Therefore, the probable subtended neural basis is the canonical neuron system (e.g. Raos et al. 1996), active during both the execution of object-directed actions and the mere observation of the same objects (Rizzolatti and Craighero 2004). Whether this system underlies only the affordance effect we found or whether it is involved in the mechanism of avoidance in act during dangerous object processing should be object of future research.

In conclusion, albeit this study is pioneer in the contest of object dangerousness representation, as well as in the contest of studies using line bisection with flanker objects, the observed results permitted us to confirm and clarify the following points. First, humans are sensitive to objects affordances, in the sense that representation of objects includes in itself the motor codes activated during the interaction with objects. This may be the case also for dangerous objects, as recently suggested also by Coello et al. (2012) who found that the dangerousness of everyday manipulable objects influenced the boundary of peripersonal space. The bias we observed is in line with the claim of sensitivity even to dangerous affordances. In this sense, our results could represent an additional contribution to the literature about the influence of emotion on domains of cognition such as attention and perception (for a review, see Dolan 2002). Second, this finding adds to previous studies on danger processing indicating that graspability is an important aspect of dangerous objects.

Future studies will shed light on the properties, and the time-course of the mechanisms of avoidance elicited by dangerous object affordances.

Acknowledgments This work was supported by grant from MIUR (PRIN 2008) and by the European Community, in FP7 project ROSSI: Emergence of Communication in RObots through Sensorimotor and Social Interaction (Grant agreement no: 216125). Part of this work was carried on with the support of the Marino Golinelli Foundation (Bologna, Italy). The authors thank Carlo Toneatto and Luisa Girelli (University of Milano-Bicocca, Italy) for a preliminary version of the task; EMCOlab group (www.emco.unibo.it) for helpful comments.

\section{References}

Anelli F, Nicoletti R, Borghi AM (2010) Categorization and action: what about object consistence? Acta Psychol 133:203-211

Anelli F, Borghi AM, Nicoletti R (2012a) Grasping the pain: motor resonance with dangerous affordances. Conscious Cogn 21:1627-1639. doi:10.1016/j.concog.2012.09.001

Anelli F, Nicoletti R, Kalkan S, Sahin E, Borghi AM (2012b) Human and robotics hand grasping danger. In: Proceedings of the International Joint Conference on Neural Networks (IJCNN) pp $1613-1620$

Avenanti A, Bueti D, Galati G, Aglioti SM (2005) Transcranial magnetic stimulation highlights the sensorimotor side of empathy for pain. Nat Neurosci 8:955-960

Avenanti A, Minio-Paluello I, Bufalari I, Aglioti SM (2006) Stimulusdriven modulation of motor-evoked potentials during observation of others' pain. NeuroImage 32:316-324

Avenanti A, Sirigu A, Aglioti SM (2010) Racial bias reduces empathic sensorimotor resonance with other-race pain. Curr Biol 20:1018-1022 
Barton JJ, Behrmann M, Black S (1998) Ocular search during line bisection: the effects of hemi-neglect and hemianopia. Brain 121:1117-1131

Bisiach E, Capitani E, Colombo A, Spinnler H (1976) Having a horizontal segment: a study on hemisphere-damaged patients with cerebral focal lesions. Schweiz Arch Neurol 118:199-206

Blanchette I (2006) Snakes, spiders, guns, and syringes: how specific are evolutionary constraints on the detection of threatening stimuli? Quart J Exp Psyc 59(8):1484-1504

Borghi AM, Flumini A, Natraj N, Wheaton LA (2012) One hand, two objects: emergence of affordance in contexts. Brain Cogn 80(1):64-73

Bub DN, Masson MEJ (2010) Grasping beer mugs: on the dynamics of alignment effects induced by handled objects. J Exp Psychol Hum Percept Perform 36:341-358

Castiello U (2003) Understanding other people's actions: intention and attention. J Exp Psychol Hum Percept Perform 29:416-430

Chao LL, Martin A (2000) Representation of manipulable man-made objects in the dorsal stream. Neuroimage 12:478-494

Coello Y, Bourgeois J, Iachini T (2012) Embodied perception of reachable space: how do we manage threatening objects? Cogn Process 13(Suppl 1):131-135

Craighero L, Fadiga L, Rizzolatti G, Umiltà CA (1998) Visuomotor priming. Vis Cogn 5:109-125

Craighero L, Fadiga L, Rizzolatti G, Umiltà CA (1999) Action for perception: a motor-visual attentional effect. J Exp Psychol Hum Percept Perform 25:1673-1692

Creem-Regehr SH, Lee JN (2005) Neural representations of graspable objects: are tools special? Cogn Brain Res 22:457-469

Dolan RJ (2002) Emotion, cognition, and behaviour. Science 298:1191-1194

Edwards MG, Humphreys GW, Castiello U (2003) Motor facilitation following action observation: a behavioural study in prehensile action. Brain Cognition 53:495-502

Ellis R, Tucker M (2000) Micro-affordance: the potentiation of components of action by seen objects. Brit J Psychol 91:451-471

Fagioli S, Hommel B, Schubotz RI (2007) Intentional control of attention: action planning primes action-related stimulus dimensions. Psychol Res 71:22-29

Fischer MH (2001) Cognition in the line bisection task. Trends Cogn Sci 5:460-462

Fischer HM, Prinz J, Lotz K (2008) Grasp cueing shows obligatory attention to action goals. Q J Exp Psychol 61:860-868

Gerlach C, Law I, Paulson OB (2002) When action turns into words. Activation of motor-based knowledge during categorization of manipulable objects. J Cognitive Neurosci 14:1230-1239

Gibson JJ (1979) The ecological approach to visual perception. Houghton Mifflin, Boston

Goslin J, Dixon T, Fischer MH, Cangelosi A, Ellis R (2012) Electrophysiological examination of embodiment in vision and action. Psychol Sci 23(2):152-157

Grafton ST, Fadiga L, Arbib MA, Rizzolatti G (1997) Premotor cortex activation during observation and naming of familiar tools. NeuroImage 6:231-236
Jewell G, McCourt ME (2000) Pseudoneglect: a review and metaanalysis of performance factors in line bisection tasks. Neuropsychologia 38:93-110

Johnson-Frey SH (2004) The neural bases of complex tool use in humans. Trends Cogn Sci 8:71-78

Kerkhoff G, Bucher L (2008) Line bisection as an early method to assess homonymous hemianopia. Cortex 44:200-205

Martin A (2007) The representation of object concepts in the brain. Ann Rev Psychol 58:25-45

Morrison I, Peelen MP, Downing P (2007a) The sight of others' pain modulates motor processing in human cingulated cortex. Cereb Cortex 17:2214-2222

Morrison I, Poliakov E, Gordon L, Downing P (2007b) Responsespecific effects of pain observation on motor behavior. Cognition 104:407-416

Öhman A, Flykt A, Esteves F (2001) Emotion drives attention: detecting the snake in the grass. J Exp Psychol Gen 130(3):466-478

Ranzini M, Girelli L (2012) Exploiting illusory effects to disclose similarities in numerical and luminance processing. Atten Percept Psychophys 74(5):1001-1008

Ranzini M, Borghi AM, Nicoletti R (2011a) With hands I do not centre! Action- and object-related effects of hand-cueing in the line bisection. Neuropsychologia 49:2918-2928

Ranzini M, Lugli L, Anelli M, Carbone R, Nicoletti R, Borghi AM (2011b) Graspable objects shape number processing. Front Hum Neurosci 5:147

Raos V, Umiltà MA, Murata A, Fogassi L, Gallese V (1996) Functional properties of grasping-related neurons in the ventral premotor area F5 of the macaque monkey. J Neurophysiol 95(2):709-729

Rizzolatti G, Craighero L (2004) The mirror-neuron system. Ann Rev Neurosci 27:169-192

Schenkenberg T, Bradford DC, Ajax ET (1980) Line bisection and unilateral visual neglect in patients with neurologic impairment. Neurology 30:509-517

Singer T, Seymour B, O'Doherty J, Kaube H, Dolan RJ, Frith CD (2004) Empathy for pain involves the affective but not sensory components of pain. Science 303:1157-1162

Symes E, Tucker M, Ellis R, Vainio L, Ottoboni G (2008) Grasp preparation improves change detection for congruent objects. J Exp Psychol Hum Percept Perform 34:854-871

Symes E, Ottoboni G, Tucker M, Ellis R, Tessari A (2010) When motor attention improves selective attention: the dissociating role of saliency. Q J Exp Psychol 63:1387-1397

Toba M-N, Cavanagh P, Bartolomeo P (2011) Attention biases the perceived midpoint of horizontal lines. Neuropsychologia 49(2):238-246

Tucker M, Ellis R (1998) On the relations between seen objects and components of potential actions. J Exp Psychol Hum Percept Perform 24:830-846

Tucker M, Ellis R (2001) The potentiation of grasp types during visual object categorization. Visual Cogn 8:769-800

Tucker M, Ellis R (2004) Action priming by briefly presented objects. Acta Psychol 116:185-203 